\title{
Imagine, believe, and achieve
}

\author{
Amalia Peix, MD, PhD, FACC, ASNC, ${ }^{a}$ and Kenia Padrón, MD $^{a}$ \\ a Nuclear Medicine Department, Institute of Cardiology, La Habana, CP, Cuba
}

Received Apr 2, 2021; accepted Apr 5, 2021

doi: $10.1007 / \mathrm{s} 12350-021-02630-w$

\section{See related article, pp. 825-830}

\section{CARDIAC AMYLOIDOSIS: NEW APPROACH TO AN UNDER-RECOGNIZED DISEASE}

Cardiac amyloidosis was an under-recognized and undertreated disease until some years ago. Multimodality imaging plays a key role in the diagnosis of this progressive infiltrative cardiomyopathy, in addition to the clinical picture, endomyocardial/fat pad biopsies, and serum cardiac biomarkers. Although echo and cardiac magnetic resonance (CMR) provide structural and functional data, but neither can provide a distinction between transthyretin amyloid cardiomyopathy (ATTR$\mathrm{CM}$ ) and light chain amyloid cardiomyopathy (AL-CM).

Among imaging techniques, scintigraphy with bone-avid tracers: technetium-99m-3,3-diphosphono1,2-propanodicarboxylic acid $\left({ }^{99 \mathrm{~m}} \mathrm{Tc}-\mathrm{DPD}\right)$ and hydroxymethylene diphosphonate $\left({ }^{99 \mathrm{~m}} \mathrm{Tc}-\mathrm{HMDP}\right){ }^{1-3}$ and technetium- $99 \mathrm{~m}$ pyrophosphate $\left({ }^{99 \mathrm{~m}} \mathrm{Tc}-\mathrm{PYP}\right)$, mainly used in Canada, US, and Latin America, has emerged as a very sensitive and specific test to diagnose transthyretin amyloidosis (ATTR). ${ }^{2,4,5}$ It makes possible to differentiate ATTR from cardiac light chain amyloidosis (AL) non-invasively, with a significant accuracy and a $100 \%$ positive predictive value, mainly when performed with adjunctive clonal analysis with free light chain immunofixation to exclude AL amyloidosis. ${ }^{4,5}$ If TTR amyloidosis is diagnosed, a genotype testing is important to differentiate between wild-type (non-mutant) (ATTRwt) or mutated transthyretin (ATTRm) (hereditary form).

Reprint requests: Amalia Peix, MD, PhD, FACC, ASNC, Nuclear Medicine Department, Institute of Cardiology, 17 No. 702, Vedado, La Habana, CP 10 400, Cuba; atpeix@gmail.com

J Nucl Cardiol 2021;28:831-4.

1071-3581/\$34.00

Copyright (C) 2021 American Society of Nuclear Cardiology.
In the consensus recommendations, the appropriate clinical scenarios where bone scintigraphy with $99 \mathrm{mTc}-$ PYP, -DPD, and -HMDP should be used are summarized: evaluation in asymptomatic TTR gene carrier, individuals $>60$ years old with low-flow low-gradient aortic stenosis, unexplained heart failure, and increased wall thickness (especially in people of African American origin), individuals with heart failure and unexplained sensorimotor neuropathy, and individuals with known or suspected familial amyloidosis. ${ }^{6}$

However, the pathway to achieve a proper monitorization of treatment as well as the evaluation of the response to the new drugs already used is not so defined. New imaging approaches considering contrast-enhanced-cardiac magnetic resonance (CE-CMR) imaging with $\mathrm{T} 1$ mapping and molecular targeted imaging with 18 fluorine $\left({ }^{18} \mathrm{~F}\right)$ florbetapir positron emission tomography (PET) potentially provide a more accurate estimation of cardiac amyloid burden compared with echocardiography, standard late gadolinium enhancement (LGE), or SPECT. Global and regional LV extracellular volume fraction (ECV) is significantly expanded in amyloid hearts as a result of amyloid infiltration. ${ }^{7,8}$ Florbetapir is a stilbene derivative that binds to AL and ATTR deposits in human hearts ex vivo and in vivo, ${ }^{9,10}$ and molecular amyloid PET makes possible the global and regional quantification of the cardiac amyloid burden.

Although the evaluation of myocardial uptake on ${ }^{99 \mathrm{~m}} \mathrm{Tc}-\mathrm{PYP}$ and ${ }^{99 \mathrm{~m}} \mathrm{Tc}-\mathrm{DPD}$ scintigraphies is based on a visual interpretation by means of Perugini scores using planar imaging, as well as a semiquantitative assessment using the heart-to-contralateral lung $(\mathrm{H} / \mathrm{CL})$ ratio with reasonable results, ${ }^{6,11}$ enough for a cardiac ATTR diagnosis, the visual and semiquantitative assessment is not able neither to properly stratify prognosis nor to assess disease progression or therapeutic response. ${ }^{12-14}$ Thus, quantification of SPECT radiotracers is also being explored using SPECT/CT images, by means of calculation of indexes such as SUVmax, SUVpeak, and SUVmax and SUVpeak normalized to bone activity 
(nSUVmax and nSUVpeak, respectively). ${ }^{12,13}$ According to this, the use of hybrid SPECT/CT systems may have an advantage over SPECT-only system, not merely for the reduction of equivocal interpretations thanks to the attenuation correction and the better definition of myocardial boundaries, but also due to the possibility of quantification. Absolute quantitative measurements also allow radiomics approaches to evaluation of cardiac amyloidosis.

An ATTR prevalence of $16 \%$ has been found among patients undergoing percutaneous aortic valve replacement for severe aortic stenosis, ${ }^{15} 13 \%$ among patients with heart failure with a preserved ejection fraction, ${ }^{16} 5 \%$ among patients with presumed hypertrophic cardiomyopathy, ${ }^{17}$ and $7 \%$ to $8 \%$ among patients with carpal tunnel syndrome. ${ }^{18}$

In this number of the Journal of Nuclear Cardiology, Bianco et al ${ }^{19}$ presented a retrospective, observational, monocentric study that included all patients who underwent a whole-body bone scintigraphy (with ${ }^{99 \mathrm{~m}} \mathrm{Tc}-\mathrm{HDP}$ or ${ }^{99 \mathrm{~m}} \mathrm{Tc}-\mathrm{DPD}$ ) for non-cardiac purposes between January 2015 and May 2020 in their center, with the aim of estimating the prevalence of findings suspicious for TTR cardiac amyloidosis. They found a $0.54 \%$ (23/4228) prevalence of Perugini score grades 2 and 3. An Australian study also looking at incidental cardiac uptake on bone scans indicated for non-cardiac purposes found a prevalence of ATTR-CM of $4.2 \%$ in men aged 85 and over, ${ }^{20}$ confirming that ATTR-CM is not as uncommon.

In their study, Bianco et al found that 14 subjects $(60.9 \%)$ had a history of arterial hypertension, 1 subject (4.4\%) had a history of coronary artery disease, $3(13 \%)$ a permanent pacemaker, and $11(48 \%)$ a previous episode of heart failure. Eight subjects presented known neuropathy (34.8\%) and 5 carpal tunnel syndrome (21.7\%), while five patients $(21.7 \%)$ were totally asymptomatic, without any sign or symptom allowing their physicians to suspect cardiac or systemic involvement of TTR amyloidosis before the bone scintigraphy. Thus, careful analysis of scintigraphic images looking for cardiac uptake (and, if this is the case, adding SPECT images) can contribute to an earlier detection and treatment of cardiac amyloidosis in a preclinical stage, potentially improving prognosis.

The true prevalence of suspected ATTR cannot be demonstrated from this study given reasons such as: the lack of SPECT imaging and genotyping, the possibility of early disease with low Perugini score or false negative scans, and the fact that $\mathrm{AL}$ amyloid was not excluded. Nevertheless, the detection of cardiac involvement in a preclinical stage represents an important finding to enhance the efficacy of novel therapies for ATTR and improve prognosis in these patients, although it requires a prompt clinical evaluation and further work up to confirm the diagnosis.

It is already demonstrated that if no monoclonal protein is detected and a diagnosis of $\mathrm{AL}$ cardiac amyloidosis is excluded, bone-avid scintigraphy alone, without myocardial biopsy, can be used to diagnose ATTR-CM. ${ }^{5}$ In addition, if ATTR-CM is identified, TTR genotyping should be performed to differentiate ATTRm from ATTRwt. ${ }^{3}$ However, in Bianco's study the patients did not undergo a comprehensive diagnostic workup in case of bone scintigraphy positivity for cardiac uptake, including serum and urinary immunofixation, dosage of serum free light chain and genetic testing. This should be done to confirm the diagnosis of amyloidosis TTR also in those cases with incidental information from suggestive findings of cardiac amyloidosis in a bone scintigraphy performed with noncardiac purposes.

Timing of imaging after radiotracer injection deserves special consideration. It varies between 1 and $3 \mathrm{~h}$. In general, ${ }^{99 \mathrm{~m}} \mathrm{Tc}-\mathrm{PYP}$ protocols use 1 or $3 \mathrm{~h}$ ' acquisitions (ref 11,22 Singh, Falk) (or even at both moments), while acquisition at $3 \mathrm{~h}$ is used with the rest of bone-avid tracers. ${ }^{2,5}$ Imaging at $1 \mathrm{~h}$ offers advantages such as more patient comfort, fast laboratory throughput, and high count images allowing the use of lower radiotracer dose. On the other hand, although imaging at $3 \mathrm{~h}$ reduces the possibility of blood pool images, it results fundamental to have SPECT images to differentiate blood pool versus myocardial uptake, which can be difficult using planar images only.

In the study of Bianco et al, SPECT imaging was not routinely acquired because the exam was performed mainly for oncology or orthopedic reasons. Thus, although the scintigraphy allowed to rise suspicions of ATTR heart involvement in one fifth of patients who were completely asymptomatic at the moment of bone scintigraphy, and this undoubtedly constitutes an important asset, SPECT images are necessary not only to distinguish between myocardial uptake and blood pool, but also to detect rib fractures and soft tissue uptake overlying the heart, all of which may potentially cause false positive scans.

Thus, an important take-home message of this work is the need of inclusion of SPECT images in those patients with cardiac uptake of Perugini grade 2 or 3 in a bone-avid radiotracer scintigraphy, which is highly suspicious for ATTR-CM, although the original indication of the exam was an oncological or orthopedic disease.

In addition, semiquantitative assessment by means of a $\mathrm{H} / \mathrm{CL}$ ratio is also important, because it offers some correction for background, blood pool, and soft tissue activity, although it does not eliminate the need of 
SPECT acquisition. Specifically for HMDP scans, Heart/Whole Body ratio (H/WB) improves on the visual score, and is more reliable than $\mathrm{H} / \mathrm{CL}$ ratio. ${ }^{11}$

\section{MULTIMODALITY ALTERNATIVES FOR THE FUTURE}

The recent advances in disease modifying treatments, namely inotersen, patisiran, and tafamidis to treat cardiac ATTR amyloidosis require a useful and applicable method to detect early disease and to monitorize the response to treatment. If the disease is detected in a preclinical stage, treatment can be started before cardiac damage is irreversible. Imaging can offer a valuable contribution to this, but it is still under investigation which technique (or combination of techniques) is the best to use.

Regarding CMR, T1 mapping and ECV represent interesting alternatives: In contrast to ECV,

which characterizes the extracellular space, native T1 provides a combined signal from myocyte and extracellular space. Native T1 is markedly increased in cardiac amyloidosis, ${ }^{21}$ with $92 \%$ sensitivity and $91 \%$ specificity to detect it. ${ }^{22}$ Although it is particularly useful in patients with cardiac amyloidosis and renal dysfunction with contraindication to gadolinium, its main limitation is the lack of reproducibility for $1.5-\mathrm{T}$ vs. 3.0-T scanners. In addition, its independent prognostic value over other risk markers has not been definitively demonstrated. ${ }^{23}$

Post-contrast T1 mapping and ECV estimation are performed post gadolinium. ECV is more reproducible than absolute $\mathrm{T} 1$ values, and it is markedly elevated in cardiac amyloidosis, but not able to differentiate between subtypes of amyloidosis. Although quantification of ECV can help to track disease burden and response to therapy, as demonstrated in $\mathrm{AL} \mathrm{CA},{ }^{24}$ more prospective studies are needed.

It has been shown that bone-avid radiotracer scintigraphy adds significant value to echocardiography and CMR because it can distinguish ATTR cardiac amyloidosis from other forms of heart diseases with left ventricular (LV) thickening. ${ }^{5}$ Nevertheless, bone-avid radiotracer images lack structural and hemodynamic information, and so they are currently used in conjunction with echocardiography or CMR. Thus, multimodality is needed to appropriately manage these patients.

In addition, another big problem is the lack of a real and effective means of assessing the response to treatment. Nowadays, LV mass by echocardiography and ECV by CMR are the current quantitative tools used to estimate cardiac amyloid burden and response to therapy. Nevertheless, newly developed SPECT technology with CT-based attenuation correction, including CZT-based detection systems with high sensitivity, making possible a reproducible quantification, may be a good option. ${ }^{13,25}$

Strongly related to the screening with bone-avid tracers' cardiac scintigraphy in asymptomatic persons at risk for ATTR amyloidosis is the idea of leveraging artificial intelligence for early detection of cardiac involvement in a susceptible population. ${ }^{26}$ Machine learning, supported by a hugh database of images and electronic health records, may contribute to better integrate imaging modalities.

\section{CONCLUSIONS}

Cardiac amyloidosis is still a growing research subject. New drugs, new imaging approaches for diagnosis, risk stratification, and assessment of response to treatment contribute to improve prognosis of this, until recently, under-recognized disease. Multicenter comparative effectiveness studies in larger populations are still needed. However, a wise use of the old bone-avid tracer scintigraphy plays a key role for cardiac ATTR diagnosis.

\section{Disclosure}

The authors have indicated that they have no financial conflict of interest.

\section{References}

1. Galat A, Rosso J, Guellich A, Van Der Gucht A, Rappeneau S, Bodez D, et al. Usefulness of (99m)Tc-HMDP scintigraphy for the etiologic diagnosis and prognosis of cardiac amyloidosis. Amyloid 2015;22:210-20.

2. Perugini E, Guidalotti PL, Salvi F, Cooke RM, Pettinato C, Riva $\mathrm{L}$, et al. Noninvasive etiologic diagnosis of cardiac amyloidosis using 99mTc-3,3-diphosphono-1,2-propanodicarboxylic acid scintigraphy. J Am Coll Cardiol 2005;46:1076-84.

3. Maurer MS, Bokhari S, Damy T, Dorbala S, Drachman BM, Fontana M, et al. Expert consensus recommendations for the suspicion and diagnosis of transthyretin cardiac amyloidosis. Circ Heart Fail 2019;12: https://doi.org/10.1161/CIRCHEARTFAI LURE.119.006075.

4. Bokhari S, Castaño A, Pozniakoff T, Deslisle S, Latif F, Maurer MS. (99m)Tc-pyrophosphate scintigraphy for differentiating lightchain cardiac amyloidosis from the transthyretin-related familial and senile cardiac amyloidoses. Circ Cardiovasc Imaging 2013;6:195-201.

5. Gillmore JD, Maurer MS, Falk RH, Merlini G, Damy T, Dispenzieri A, et al. Nonbiopsy diagnosis of cardiac transthyretin amyloidosis. Circulation 2016;133:2404-12.

6. Dorbala S, Ando Y, Bokhari S, Dispenzieri A, Falk RH, Ferrari VA, et al. ASNC/AHA/ASE/EANM/HFSA/ISA/SCMR/SNMMI expert consensus recommendations for multimodality imaging in 
cardiac amyloidosis: Part 1 of 2-evidence base and standardized methods of imaging. J Nucl Cardiol 2019;26:2065-123.

7. Fontana M, Pica S, Reant P, Abdel-Gadir A, Treibel TA, Banypersad SM, et al. Prognostic value of late gadolinium enhancement cardiovascular magnetic resonance in cardiac amyloidosis. Circulation 2015;132:1570-9.

8. Fontana M, Banypersad SM, Treibel TA, Abdel-Gadir A, Maestrini V, Lane T, et al. Differential myocyte responses in patients with cardiac transthyretin amyloidosis and light-chain amyloidosis: A cardiac MR imaging study. Radiology 2015;277:388-97.

9. Dorbala S, Vangala D, Semer J, Strader C, Bruyere JR, Di Carli MF, et al. Imaging cardiac amyloidosis: A pilot study using (18)Fflorbetapir positron emission tomography. Eur J Nucl Med Mol Imaging 2014;41:1652-62.

10. Park MA, Padera RF, Belanger A, Dubey S, Hwang DH, Veeranna $\mathrm{V}$, et al. 18F-Florbetapir binds specifically to myocardial light chain and transthyretin amyloid deposits: autoradiography study. Circ Cardiovasc Imaging 2015. https://doi.org/10.1161/circimagi ng.114.002954.

11. Gallini C, Tutino F, Martone R, Ciaccio A, Costanzo EN, Taborchi $\mathrm{G}$, et al. Semi-quantitative indices of cardiac uptake in patients with suspected cardiac amyloidosis undergoing 99mTc- HMDP scintigraphy. J Nucl Cardiol 2021;28:90-9.

12. Caobelli F, Braun M, Haaf P, Wild D, Zellweger MJ. Quantitative 99mTc-DPD SPECT/CT in patients with suspected ATTR cardiac amyloidosis: Feasibility and correlation with visual scores. J Nucl Cardiol 2020;27:1456-63.

13. Dorbala S, Park MA, Cuddy S, Singh V, Sullivan K, Kim S, et al. Absolute quantitation of cardiac $99 \mathrm{mTc}$-pyrophophate using Cadmium Zinc Telluride-based SPECT/CT. J Nucl Med 2020. h ttps://doi.org/10.2967/jnumed.120.247312.

14. Dorbala S, Kijewski MF, Park MA. Quantitative bone-avid tracer SPECT/CT for cardiac amyloidosis: A crucial step forward. J Am Coll Cardiol 2020;13:1364-7.

15. Castaño A, Narotsky DL, Hamid N, Khalique OK, Morgenstern R, DeLuca A, et al. Unveiling transthyretin cardiac amyloidosis and its predictors among elderly patients with severe aortic stenosis undergoing transcatheter aortic valve replacement. Eur Heart J 2017;38:2879-87.

16. González-López E, Gallego-Delgado M, Guzzo-Merello G, de Haro-Del Moral FJ, Cobo-Marcos M, Robles C, et al. Wild-type transthyretin amyloidosis as a cause of heart failure with preserved ejection fraction. Eur Heart J 2015;36:2585-94.

17. Damy T, Costes B, Hagège AA, Donal E, Eicher JC, Slama M, et al. Prevalence and clinical phenotype of hereditary transthyretin amyloid cardiomyopathy in patients with increased left ventricular wall thickness. Eur Heart J 2016;37:1826-34.

18. Sperry BW, Reyes BA, Ikram A, Donnelly JP, Phelan D, Jaber WA, et al. Tenosynovial and cardiac amyloidosis in patients undergoing carpal tunnel release. J Am Coll Cardiol 2018;72:2040-50

19. Bianco M, Parente A, Biolè C, Righetti C, Spirito A, Luciano A, et al. The prevalence of TTR cardiac amyloidosis among patients undergoing bone scintigraphy. J Nucl Cardiol 2021. https://doi. org/10.1007/s12350-021-02575-0.

20. Cuscaden C, Smith J, Goodwin B, Ramsay S. Prevalence of transthyretin (ATTR) cardiac amyloidosis in the Australian population estimated using 99mTc-hydroxymethylene diphosphonate planar bone scans. Int Med J 2019;49:6.

21. Fontana M, Banypersad SM, Treibel TA, et al. Native T1 mapping in transthyretin amyloidosis. J Am Coll Cardiol Image. 2014;7:157-65.

22. Karamitsos TD, Piechnik SK, Banypersad SM, et al. Noncontrast T1 mapping for the diagnosis of cardiac amyloidosis. J Am Coll Cardiol Image. 2013;6:488-97.

23. Maceira AM, Prasad SK, Hawkins PN, Roughton M, Pennell DJ. Cardiovascular magnetic resonance and prognosis in cardiac amyloidosis. J Cardiovasc Magn Reson. 2008;10:54.

24. Martinez-Naharro A, Abdel-Gadir A, Treibel TA, Zumbo G, Knight DS, Rosmini S, et al. CMR-verified regression of cardiac AL amyloid after chemotherapy. J Am Coll Cardiol Image 2018;11:152-4.

25. Hutton BF, Erlandsson K, Thielemans K. Advances in clinical molecular imaging instrumentation. Clin. Transl. Imaging 2018;6:31-45

26. Khor YM, Cuddy S, Falk RH, Dorbala S. Multimodality imaging in the evaluation and management of cardiac amyloidosis. Semin Nucl Med 2020;50:295-310.

Publisher's Note Springer Nature remains neutral with regard to jurisdictional claims in published maps and institutional affiliations. 